Correspondence

Helena Santos

santos@itgb.unl.pt
Received 28 June 2006

Revised 15 September 2006

Accepted 19 September 2006

\section{Biochemical and genetic characterization of the pathways for trehalose metabolism in Propionibacterium freudenreichii, and their role in stress response}

\author{
Filipa S. Cardoso, Rute F. Castro, Nuno Borges and Helena Santos \\ Instituto de Tecnologia Química e Biológica, Universidade Nova de Lisboa and Instituto de \\ Biologia Experimental e Tecnológica, Rua da Quinta Grande, 6, Apt. 127, 2780-156 Oeiras, \\ Portugal
}

Propionibacterium freudenreichii accumulates high levels of trehalose, especially in response to stress. The pathways for trehalose metabolism were characterized, and their roles in response to osmotic, oxidative and acid stress were studied. Two pathways were identified: the trehalose-6-phosphate synthase/phosphatase (OtsA-OtsB) pathway, and the trehalose synthase (TreS) pathway. The former was used for trehalose synthesis, whereas the latter is proposed to operate in trehalose degradation. The activities of OtsA, OtsB and TreS were detected in cell extracts; the corresponding genes were identified, and the recombinant proteins were characterized in detail. In crude extracts of $P$. freudenreichii, OtsA was specific for ADP-glucose, in contrast to the pure recombinant OtsA, which used UDP-, GDP- and TDP-glucose, in addition to ADP-glucose. Moreover, the substrate specificity of OtsA in cell extracts was lost during purification, and the recombinant OtsA became specific to ADP-glucose upon incubation with a dialysed cell extract. The level of OtsA was enhanced (approximately twofold) by osmotic, oxidative and acid stress, whereas the level of TreS remained constant, or it decreased, under identical stress conditions. Therefore, the OtsA-OtsB pathway plays an important role in the synthesis of trehalose in response to stress. It is most likely that trehalose degradation proceeds via TreS to yield maltose, which is subsequently catabolized via amylomaltase activity. Hydrolytic activities that are potentially involved in trehalose degradation (trehalase, trehalose phosphorylase,

trehalose-6-phosphate phosphorylase and trehalose-6-phosphate hydrolase) were not present. The role of trehalose as a common response to three distinct stresses is discussed.

\section{INTRODUCTION}

Trehalose is widely distributed in living cells, where it plays a variety of roles generally associated with protection against stress (Argüelles, 2000; Elbein et al., 2003). This disaccharide is frequently found in yeast, fungi and plants, but it also occurs in many bacteria, and several halophilic or hyperthermophilic archaea (da Costa et al., 1998; Santos \& da Costa, 2001; Elbein et al., 2003).

At least four pathways for the synthesis of trehalose have been reported thus far: (i) the OtsA-OtsB pathway, the most common route, involves the transfer of glucose from UDPglucose to glucose 6-phosphate to yield trehalose 6phosphate, which is subsequently converted to trehalose (Brüggemann et al., 2004); (ii) the TreS pathway, a reversible

The GenBank/EMBL/DDBJ accession numbers for the nucleotide sequence of the DNA fragment containing the ots $A$ and ots $B$ genes, and the sequence containing the treS gene, are DQ356268 and D0356269, respectively. pathway involving the intramolecular rearrangement of the $\alpha-(1 \rightarrow 4)$ glycosidic bond of maltose into an $\alpha(1 \rightarrow 1)$ glycosidic bond to yield trehalose (Nishimoto et al., 1996); (iii) the TreY-TreZ pathway, in which the terminal $\alpha(1 \rightarrow 4)$ bond of an $\alpha(1 \rightarrow 4)$-glucan polymer is converted into an $\alpha(1 \rightarrow 1)$ bond via transglycosylation, and subsequently the terminal disaccharide is cleaved to release trehalose (Nakada et al., 1995); and (iv) a single-step pathway involving trehalose glycosyltransferring synthase, a recently discovered enzyme that catalyses the reversible conversion of glucose and NDP-glucose into trehalose $(\mathrm{Qu}$ et al., 2004).

In many organisms, trehalose catabolism proceeds via trehalase, resulting in the formation of two glucose molecules (Horlacher et al., 1996; Elbein et al., 2003). Other enzymes for hydrolysis of trehalose or trehalose 6phosphate have been described: trehalose phosphorylase catalyses the phosphorolysis of trehalose producing $\beta$ glucose 1-phosphate and glucose; trehalose-6-phosphate 
phosphorylase converts trehalose 6-phosphate into $\beta$ glucose 1-phosphate and glucose 6-phosphate; and, finally, trehalose-6-phosphate hydrolase produces glucose and glucose 6-phosphate from trehalose 6-phosphate (Belocopitow \& Maréchal, 1970; Rimmele \& Boos, 1994; Andersson et al., 2001).

Dairy propionibacteria are important organisms in cheesemaking, and in the production of other dairy products. In addition, the potential probiotic properties of some species (Propionibacterium acidipropionici, Propionibacterium freudenreichii, Propionibacterium jensenii and Propionibacterium thoenii) have been suggested in recent years (Jan et al., 2002; Huang \& Adams, 2004). The presence of trehalose in propionibacteria has been known for nearly five decades (Stjernholm, 1958); however, trehalose metabolism has not been studied in any member of the genus Propionibacterium.

A few years ago, we showed that trehalose is widespread within the genus Propionibacterium (Cardoso et al., 2004). In particular, $P$. freudenreichii subsp. shermanii strain NIZO B365 accumulates trehalose to remarkable levels, and the trehalose content increases considerably in response to osmotic, oxidative and acid stress [up to $40 \%(\mathrm{w} / \mathrm{w})$ of the cell protein]. These findings triggered our interest in elucidating trehalose metabolism and regulation in $P$. freudenreichii under different stressful conditions. Given the importance of trehalose as a preservative of food flavour, and in cell protection against different stresses, knowledge of trehalose metabolism could be of importance in directing strain manipulation: for example, in improving acid resistance, and allowing extended fermentation.

In the present work, the pathways for synthesis and degradation of trehalose were investigated. The OtsAOtsB and TreS pathways were genetically and biochemically characterized. Additionally, the levels of OtsA and TreS were examined under different growth conditions by immunoassays to study the role of the two pathways, and their regulatory properties under osmotic, oxidative and acid stress.

\section{METHODS}

P. freudenreichii and growth conditions. P. freudenreichii subsp. shermanii strain NIZO B365 [Netherlands Institute for Dairy Research (NIZO), Ede, The Netherlands] was grown in medium buffered with potassium phosphate, in 21 fermenters, under controlled conditions of $\mathrm{pH}(7.0)$ and temperature $\left(30^{\circ} \mathrm{C}\right)$, as previously described (Cardoso et al., 2004). For enzymic assays, and for purification of native OtsA, cells were grown under osmotic stress $[1.5 \%$ $(w / v) \mathrm{NaCl}]$ at $\mathrm{pH} 5.5$.

Preparation of $\boldsymbol{P}$. freudenreichii cell extracts. Cells were harvested during the exponential phase of growth, and suspended in PIPES buffer ( $50 \mathrm{mM}, \mathrm{pH} 6.5$ ), containing $2 \mathrm{mM}$ DTT and a mixture of protease inhibitors: $100 \mu \mathrm{M}$ PMSF, $10 \mu \mathrm{M}$ leupeptin, and $10 \mu \mathrm{M}$ antipain. The cells were disrupted in a French press, and cell debris was removed by centrifugation. For enzymic assays, lowmolecular-mass compounds were removed in a PD-10 column (Amersham Biosciences); for the purification of native OtsA, the extract was dialysed twice (membrane cut-off $12-14 \mathrm{kDa}$ ) against Tris/HCl buffer $(20 \mathrm{mM}, \mathrm{pH}$ 7.6). The amount of protein was estimated by using the BCA protein assay kit (Pierce).

Enzyme assays. Activity of OtsA was determined in a reaction mixture containing acetate buffer $(50 \mathrm{mM}, \mathrm{pH} 5.5), 10 \mathrm{mM} \mathrm{MgCl}$, $10 \mathrm{mM}$ glucose 6-phosphate, and $5 \mathrm{mM}$ NDP-glucose (in which $\mathrm{N}$ represents A, U, G or T). The activity of OtsA using CDP-glucose was not studied because this substrate is not commercially available. The reaction was initiated with the enzyme $(0.8 \mu \mathrm{g})$, then incubated at $37^{\circ} \mathrm{C}$ for different periods of time, and stopped by incubation in boiling water for $5 \mathrm{~min}$. Afterwards, the reaction mixture was incubated for $30 \mathrm{~min}$ at $37^{\circ} \mathrm{C}$ with $1 \mu \mathrm{g}$ recombinant OtsB. The $\mathrm{Pi}$ released was determined using the spectrophotometric method described by Ames (1966), after confirming that OtsB was specific for trehalose 6-phosphate. In cell extracts, or during the purification of native OtsA, trehalose formation was detected by TLC after treatment with alkaline phosphatase. The OtsB activity was assayed in a reaction mixture containing MES buffer $(50 \mathrm{mM}, \mathrm{pH} 6.5), 10 \mathrm{mM}$ $\mathrm{MgCl}_{2}$, and $5 \mathrm{mM}$ trehalose 6-phosphate. The reaction was initiated with the enzyme $(0.3 \mu \mathrm{g})$, then incubated at $37^{\circ} \mathrm{C}$ for different periods of time, and stopped by freezing in liquid nitrogen. Pi was determined as described above.

The biochemical characterization of TreS was performed in a reaction mixture containing phosphate buffer (50 mM, pH 5.2) and $5 \mathrm{mM}$ maltose. The reaction was started with the enzyme $(1 \mu \mathrm{g})$, then incubated at $37^{\circ} \mathrm{C}$ for different periods of time, and stopped by incubation in boiling water for $5 \mathrm{~min}$. The same conditions were used to study the reverse reaction, except that $5 \mathrm{mM}$ trehalose was used instead of maltose. The reaction product (trehalose or maltose) was incubated for $1 \mathrm{~h}$ at $50{ }^{\circ} \mathrm{C}$ with $30 \mu \mathrm{g}$ trehalase (isolated from Rhodothermus marinus) or $0.1 \mathrm{U}$ maltase (Sigma), respectively, for $1 \mathrm{~h}$ at $37^{\circ} \mathrm{C}$. Commercially available trehalase was not suitable because it contains $\alpha$-glucosidase as a contaminant. The formation of glucose was determined enzymically (D-glucose kit; Boehringer Mannheim). In cell extracts, the samples were incubated for $1 \mathrm{~h}$ at $37^{\circ} \mathrm{C}$, and the formation of trehalose or maltose was evaluated by TLC. Whenever necessary, the assignment of spots was confirmed by treatment with trehalase or maltase; moreover, the reaction products were analysed in separate runs using two solvents in order to optimize spot identification.

The activity of amylomaltase was investigated in reaction mixtures containing sodium phosphate buffer $(25 \mathrm{mM}$, pH 6.5$), 5 \mathrm{mM}$ maltoheptaose, maltopentaose, maltotetraose or maltotriose (Sigma), and an appropriate amount of enzyme. The reaction mixtures were incubated at $37^{\circ} \mathrm{C}$ for $0-24 \mathrm{~h}$, and the reactions were stopped by incubation in boiling water for $5 \mathrm{~min}$. The products were analysed by TLC.

The activities of the TreY-TreZ pathway were investigated as described by Maruta et al. (1995). Trehalose phosphorylase was examined as described by Maréchal \& Belocopitow (1972). The activity of trehalose glycosyltransferring synthase was tested in both directions, as reported by Qu et al. (2004). Assays to investigate the presence of trehalase in cell extracts were performed as described by Uhland et al. (2000). The activity of trehalose-6-phosphate phosphorylase and trehalose-6phosphate hydrolase was assessed by the methods described by Andersson et al. (2001) and Rimmele \& Boos (1994), respectively. In all cases, incubations were performed at $37^{\circ} \mathrm{C}$ for $1 \mathrm{~h}$, and end-product formation was analysed by TLC.

TLC conditions. TLC was performed on silica gel 60 plates (Merck). Butanol/pyridine/water (7:3:1, by vol.) or butanol/ethanol/water $(5: 3: 2$, by vol.) was used as the solvent system. The former solvent system distinguishes trehalose from maltose, while 
the latter has the advantage of separating phosphorylated compounds. Spots were visualized by spraying with $\alpha$-naphtholsulfuric acid solution, followed by charring at $120^{\circ} \mathrm{C}$.

Identification of otsA, otsB and treS genes. Chromosomal DNA from $P$. freudenreichii was isolated by standard procedures (Sambrook et al., 1989). Degenerate primers, based on two conserved regions (FFLHIPFP and GMNLVAKE) of known OtsA proteins, and on two conserved regions (AVFYEV and GDEIGM) of known TreS proteins, were designed to amplify ots $A$ and treS, respectively. Single PCR products of $595 \mathrm{bp}$ (for otsA) and $1111 \mathrm{bp}$ (for treS) were amplified, cloned into pGEM-T, and sequenced (AGOWA, Berlin, Germany). The complete sequences of otsA and treS were obtained by inverse PCR. For this, P. freudenreichii genomic DNA was partially digested with SacI, recircularized, and used to perform inverse PCR. Single PCR products of $4 \mathrm{~kb}$ (for otsA) and $2.5 \mathrm{~kb}$ (for treS) were obtained, cloned into pGEM-T, and sequenced (STAB-VIDA, Oeiras, Portugal).

Cloning and overexpression of ots $A$, ots $B$ and tres genes. The ots $A$ and ots $B$ genes were amplified by PCR using the GC-Rich PCR System Kit (Amersham Biosciences), and cloned separately in the pGEX-4T-2 plasmid between the XmaI-XhoI sites and the BamHIEcoRI sites, respectively. The treS gene was cloned in the pET-19b plasmid between the NdeI-NdeI sites. The resulting plasmids were designated pOtsA, pOtsB and pTreS. Cloning methodology followed standard protocols (Sambrook et al., 1989). The plasmids pOtsA and pOtsB were separately introduced into Escherichia coli JM107, and the plasmid pTreS was introduced into E. coli BL21(DE3). E. coli strains were grown at $37^{\circ} \mathrm{C}$ in LB medium, supplemented with ampicillin $\left(100 \mu \mathrm{g} \mathrm{ml}^{-1}\right)$, to an $\mathrm{OD}_{600}$ of 0.6 , and induced with $1 \mathrm{mM}$ IPTG for $3 \mathrm{~h}$. No inducer was added for otsB expression.

Purification of recombinant proteins. E. coli cells were collected by centrifugation, and suspended in PBS buffer $\mathrm{pH} 7.3$ (for E. coli pOtsA and pOtsB), or in His-Trap binding buffer (for E. coli pTreS). Cells were disrupted in a French press, and cell debris was removed by centrifugation. OtsA and OtsB were purified in two chromatographic steps using a GST Prep FF column and a Mono Q column, after digesting the fusion proteins with thrombin $(12 \mathrm{~h}$ at $\left.22^{\circ} \mathrm{C}\right)$. TreS was purified in a His-Trap column, and the histidine tag was cleaved with enterokinase $\left(18 \mathrm{~h}\right.$ at $\left.37^{\circ} \mathrm{C}\right)$. Single bands of 55,30 and $69 \mathrm{kDa}$ were observed in SDS-PAGE gel, corresponding to OtsA, OtsB and TreS, respectively.

\section{Purification of native trehalose-6-phosphate synthase}

Native OtsA was partially purified by FPLC (Amersham Biosciences).

Ammonium sulfate precipitation. The cell extract was treated with ammonium sulfate $(50 \%)$ at $4{ }^{\circ} \mathrm{C}$. The precipitate was dissolved in $20 \mathrm{mM}$ Tris/HCl (pH 7.6) containing $1 \mathrm{mM}$ EDTA and $5 \mathrm{mM} \beta$ mercaptoethanol, and dialysed overnight.

Ion-exchange chromatography. The dialysed sample was applied to a DEAE-Sepharose column $(20 \mathrm{mM}$ Tris/HCl, $\mathrm{pH}$ 7.6), and eluted with a linear gradient $0-1 \mathrm{M} \mathrm{NaCl}$. OtsA activity was detected at around $0.75 \mathrm{M} \mathrm{NaCl}$. After desalting, active fractions were loaded onto a Q-Sepharose column (20 mM Tris/HCl, $\mathrm{pH}$ 7.6). The enzyme was eluted around $0.5 \mathrm{M} \mathrm{NaCl}$. The dialysed active fractions were applied onto a Resource Q column (20 mM Tris/HCl, pH 7.0). OtsA activity was eluted at $0.3 \mathrm{M} \mathrm{NaCl}$.

Hydrophobic chromatography. Solid ammonium sulfate was added slowly to the active fractions to a final concentration of $0.9 \mathrm{M}$. The sample was applied to a phenyl-Sepharose column, and eluted with a linear gradient of ammonium sulfate $(0.9-0 \mathrm{M})$. OtsA activity was detected at $0.15 \mathrm{M}$ ammonium sulfate.
Ion-exchange chromatography. After dialysis, active fractions were applied onto a Mono Q column (20 mM Tris/ $\mathrm{HCl}, \mathrm{pH}$ 7.0), and the OtsA activity was detected at $0.3 \mathrm{M} \mathrm{NaCl}$.

Characterization of recombinant enzymes. OtsA substrate specificity was studied by combining a sugar acceptor unit (glucose 6-phosphate, trehalose 6-phosphate, fructose 6-phosphate, mannose 6-phosphate, galactose 6-phosphate, glucosamine 6-phosphate, ribose 5-phosphate, nitrophenyl phosphate, glucose 1-phosphate, fructose 1-phosphate, mannose 1-phosphate, galactose 1-phosphate, glucosamine 1-phosphate, glucose, fructose, maltose, lactose or galactose) with one of the four glucosyl donor substrates (ADP-, UDP-, GDP- or TDP-glucose). All the phosphate sugars mentioned above were also examined as possible substrates for OtsB. The temperature profiles for activity of OtsA, TreS and OtsB were studied between 30 and $70^{\circ} \mathrm{C}$ at $\mathrm{pH} 5.2$ (OtsA and TreS) or pH 6.5 (OtsB). The $\mathrm{pH}$ profiles of OtsA and OtsB were determined at $37^{\circ} \mathrm{C}$ using $50 \mathrm{mM}$ acetate buffer in the $\mathrm{pH}$ range 4.5-5.5, $50 \mathrm{mM}$ MES buffer in the $\mathrm{pH}$ range $5.5-7.0$, and $50 \mathrm{mM}$ BisTris propane/ $\mathrm{HCl}$ buffer in the $\mathrm{pH}$ range $6.5-8.5$. The effect of $\mathrm{pH}$ on TreS was determined at $37^{\circ} \mathrm{C}$ using $50 \mathrm{mM}$ phosphate buffer in the $\mathrm{pH}$ range $5.0-7.5$, and $50 \mathrm{mM}$ acetate buffer in the $\mathrm{pH}$ range $4.0-5.5$. Kinetic parameters $\left(V_{\max }\right.$ and $K_{\mathrm{m}}$ ) were determined under optimum conditions, using Lineweaver-Burk plots. The effect of $\mathrm{Mg}^{2+}, \mathrm{Mn}^{2+}, \mathrm{Ca}^{2+}, \mathrm{Co}^{2+}$, $\mathrm{Cu}^{2+}(10 \mathrm{mM})$ and $\mathrm{NaCl}(100 \mathrm{mM})$ was tested on OtsA, OtsB and

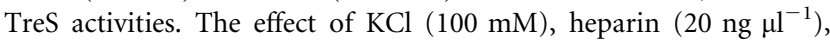
pure recombinant OtsB $\left(0.02 \mu \mathrm{g}\right.$ protein $\left.\mu \mathrm{l}^{-1}\right)$, and a dialysed extract of $P$. freudenreichii $\left(1 \mu \mathrm{g}\right.$ total protein $\left.\mu \mathrm{l}^{-1}\right)$, on OtsA activity was also examined. Moreover, the effect of $\mathrm{NaF}(10 \mathrm{mM})$ and EDTA $(10 \mathrm{mM})$ on the activity of OtsB was also studied. The effect of EDTA $(10 \mathrm{mM})$ and Tris/ $\mathrm{HCl}$ buffer $(10 \mathrm{mM})$ on TreS activity was evaluated. All the reactions were done at least in duplicate, and most were done in triplicate.

Levels of enzymes and trehalose produced in response to osmotic, oxidative and acid stress. $P$. freudenreichii cells were grown under controlled conditions of $\mathrm{pH}$ and temperature. The medium was gassed with argon during $1 \mathrm{~h}$ preceding inoculation, and the cells were allowed to grow until the mid-exponential phase. Osmotic, oxidative and acid stress were imposed in separate experiments by adding a $\mathrm{NaCl}$ solution to obtain a final concentration of $2 \%(\mathrm{w} / \mathrm{v}) \mathrm{NaCl}$, bubbling air through the culture to a final oxygen concentration of $0.115 \mathrm{mM}$, and shifting the $\mathrm{pH}$ to 5.0 with $5 \mathrm{M}$ $\mathrm{HCl}$, respectively. The cultures were allowed to grow further for $10 \mathrm{~h}$ under stress conditions. Samples were withdrawn at 0, 2, 5 and $10 \mathrm{~h}$, and used to determine protein levels (OtsA and TreS) by immunoblotting, and intracellular trehalose by ${ }^{1} \mathrm{H}-\mathrm{NMR}$ (Cardoso et al., 2004).

Western blot analyses. P. freudenreichii ( $5 \mu \mathrm{g}$ of total protein) was suspended in potassium phosphate buffer (50 mM, pH 7.0) containing $2 \%(\mathrm{w} / \mathrm{v})$ SDS. Cells were disrupted with glass beads $(<106 \mathrm{~mm}$ diameter), and the cell debris was removed by centrifugation. The rabbit primary antibodies (anti-OtsA and anti-TreS) were produced at Eurogentec (Belgium), and further purified for the immunoassays (Smith \& Fisher, 1984). The resulting anti-OtsA and anti-TreS specifically recognized the OtsA and TreS in P. freudenreichii cell extracts. After SDS-PAGE, the proteins were transferred onto PVDF membranes (Millipore), which were incubated overnight with primary antibodies. Binding was detected with the enhanced chemiluminescence system (Amersham Biosciences) after treatment with anti-rabbit antibodies conjugated with horseradish peroxidase (Sigma). The signals were detected with X-ray film, and scanned and quantified by using a program written in MatLab (MathWorks). The experiments were performed at least in triplicate. 


\section{RESULTS}

\section{Enzyme activities involved in trehalose metabolism}

The genome sequence of $P$. freudenreichii is not available; hence, the enzyme activities implicated in known pathways for the synthesis or degradation of trehalose were sought in dialysed extracts of $P$. freudenreichii. OtsA activity was assayed using glucose 6-phosphate, and ADP-, GDP-, TDPor UDP-glucose, as the substrates. ADP-glucose was the only glucose donor used to synthesize trehalose 6phosphate. The identification of this metabolite was confirmed by the detection of trehalose upon treatment of the final products with alkaline phosphatase (Fig. 1a). Phosphatase activity able to dephosphorylate trehalose 6phosphate was also detected in cell extracts.

TreS activity was detected in both directions using TLC for visualization of substrate consumption and product formation (Fig. 1b). The weak spot of glucose detected when maltose was the substrate was attributed to the presence of a low activity of $\alpha$-glucosidase (Fig. 1b). Activities potentially involved in maltose catabolism were also examined. We looked for maltooligosaccharide hydrolytic activities. Amylomaltase was detected: $1 \mathrm{~h}$ incubation of G7 with extracts of $P$. freudenreichii led to the formation of G8 and G6 (weak bands), and G4 (data not shown). For longer periods of incubation, G8, G6, G5, G4, G3, G2 and glucose were clearly detected (Fig. 1c). Spots due to formation of multiple maltooligosaccharides were also observed with G5, G4 and G3, but not with maltose. However, when maltose was incubated with trace amounts of G3 or G5, longer oligosaccharides, as well as glucose, were detected (data not shown). The activities of other enzymes potentially involved in trehalose metabolism, such as maltooligosyl trehalose synthase and trehalohydrolase, trehalose glycosyltransferring synthase, trehalase, trehalose-6-phosphate hydrolase and trehalose-6-phosphate phosphorylase, were also sought in dialysed cell extracts of $P$. freudenreichii. No evidence was found for the presence of these enzymes.

\section{Identification of ots $A$, ots $B$ and treS genes}

PCR products of 4 and $2.5 \mathrm{~kb}$ were amplified by inverse PCR using primers based on otsA and treS genes, respectively, as described in Methods. The nucleotide sequence of the $4 \mathrm{~kb}$ fragment revealed an ORF encoding a protein with 493 aa that had high identity with known OtsA proteins (Table 2). At $152 \mathrm{bp}$ upstream of the otsA gene, a second ORF was found encoding a protein with 288 aa, with considerable identity with known OtsB proteins (Table 2), and downstream of the otsA gene, a fragment (518 bp) of a third ORF was encountered, which corresponds to part of the gene $c b i B$ encoding cobinamide synthase (Fig. 2a), an enzyme involved in the synthesis of vitamin $\mathrm{B}_{12}$ (Roessner et al., 2002). Sequencing of the $2.5 \mathrm{~kb}$ fragment showed an ORF encoding a protein of 605 aa, with at least $58 \%$ identity with known TreS proteins (Table 2, Fig. 2b). Downstream of the treS gene there was an ORF with $37 \%$ identity with a hypothetical protein involved in trehalose synthesis in Propionibacterium acnes (YP_055818), and $28 \%$ identity with maltokinase from Actinoplanes missouriensis (AAQ_01690). Another ORF located upstream of the treS gene was found to have high homology (49\%) with $\alpha$ amylase from P. acnes (YP_055820).

\section{Characterization of recombinant enzymes}

We deemed it interesting to characterize the enzymes involved in trehalose metabolism of $P$. freudenreichii. For (a)

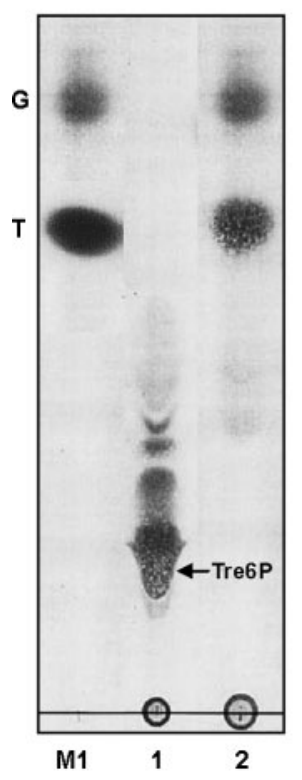

(b)

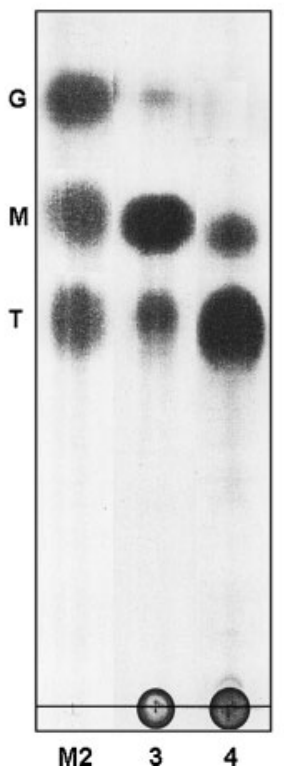

(c)

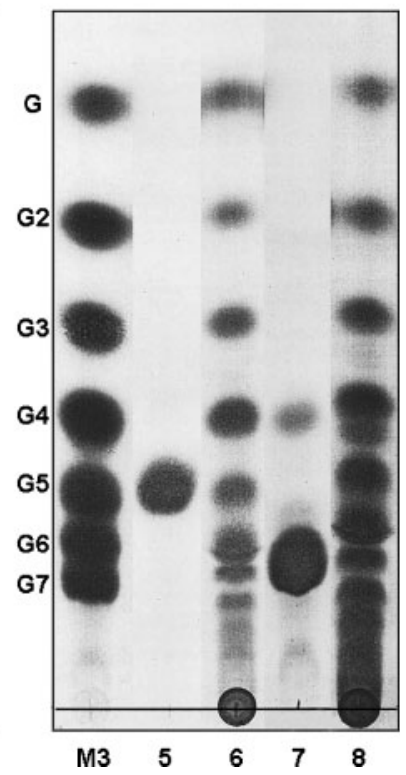

Fig. 1. Identification of enzymic activities in cell extracts by TLC analysis of reaction products. (a) Cell extract incubated with ADPglucose and glucose 6-phosphate before (lane 1) and after (lane 2) treatment with alkaline phosphatase (solvent system butanol/ethanol/water); (b) cell extract incubated with maltose (lane 3) and trehalose (lane 4) (solvent system butanol/pyridine/water); (c) cell extract incubated with maltopentaose (G5) for 0-8 h (lanes 5 and 6) and maltoheptaose (G7) (lanes 7 and 8) (solvent system butanol/pyridine/water). M1, M2 and $\mathrm{M} 3$ are standards; $\mathrm{G}$, glucose; $\mathrm{T}$, trehalose; $M$, maltose; G2-G7, maltooligosaccharides. 
(a)

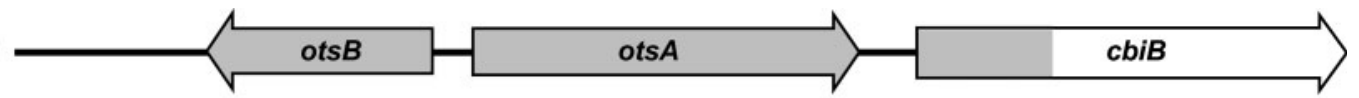

(b)

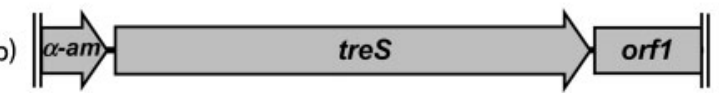

$0.5 \mathrm{~kb}$

Fig. 2. Genomic organization and flanking regions of the ots $A$-ots $B$ operon-like structure (a), and the tre $S$ gene (b). cbiB, gene encoding cobinamide synthase; $\alpha$-am, gene encoding $\alpha$-amylase; orf1, gene encoding a hypothetical protein. The nucleotide sequences identified in this work are indicated in grey. The sequence of $732 \mathrm{bp}$ upstream of ots $B$ was also obtained.

this purpose, the ots $A$, ots $B$ and treS genes were separately cloned and overexpressed in $E$. coli, and the respective proteins were purified.

Trehalose-6-phosphate synthase. The recombinant OtsA showed an absolute specificity for glucose 6-phosphate as a glucosyl acceptor. In contrast, the enzyme used ADP-glucose, UDP-glucose, GDP-glucose or TDP-glucose as glucosyl donor substrates. OtsA was not strictly dependent on $\mathrm{Mg}^{2+}$, but the presence of $10 \mathrm{mM} \mathrm{Mg}^{2+}$ was required for maximal activity. $\mathrm{Co}^{2+}$ had no effect on the activity, while other divalent cations had an inhibitory effect (Table 1). An increase in the concentration of $\mathrm{NaCl}$ from 0 to $100 \mathrm{mM}$ led to a decrease in the OtsA activity by more than $35 \%$ when ADP-glucose or TDP-glucose was the substrate. In contrast, when we used UDP-glucose or GDP-glucose, the OtsA activity was increased by 10-30\% (Table 1). Upon an increase in the $\mathrm{KCl}$ concentration from 0 to $100 \mathrm{mM}$, the OtsA activity decreased by more than $40 \%$, whereas it was not significantly affected when UDP or GDP-glucose was used as the substrate (Table 1). A strong activation of OtsA (62\%) was observed with heparin when UDP-glucose was the substrate, but no significant activation was observed with the other glucosyl donors examined (data not shown).

The maximum activity of OtsA was detected at $\mathrm{pH} 5.5$ for GDP-glucose, and pH 5.25 for ADP-glucose, UDP-glucose and TDP-glucose (Fig. 3). In this range of $\mathrm{pH}$, maximal specific activity of OtsA was determined with TDPglucose $\left(30.0 \mu \mathrm{mol} \mathrm{min}{ }^{-1} \mathrm{mg}^{-1}\right)$, followed by UDP-glucose $\left(16.8 \mu \mathrm{mol} \mathrm{min}{ }^{-1} \mathrm{mg}^{-1}\right)$, ADP-glucose $\quad(14.2 \mu \mathrm{mol}$ $\left.\mathrm{min}^{-1} \mathrm{mg}^{-1}\right)$ and GDP-glucose $\left(13.6 \mu \mathrm{mol} \mathrm{min}{ }^{-1} \mathrm{mg}^{-1}\right)$. The temperature profiles for activity were very similar for the four glucosyl donors, and maximal activity was observed at $45^{\circ} \mathrm{C}$ (data not shown). A typical Michaelis-Menten behaviour was observed for all the NDP-glucose substrates. OtsA had a higher affinity for UDP-glucose $\left(K_{\mathrm{m}}\right.$ value of $0.3 \mathrm{mM}$ ), but the maximal specific activity was reached when TDP-glucose was used (Table 1).

Table 1. Kinetic parameters and biochemical properties of recombinant OtsA, OtsB and TreS from P. freudenreichii

Activities in the presence of divalent cations, and $\mathrm{NaCl}$ and $\mathrm{KCl}$, are expressed as percentages of the maximum activity; the values are the means of at least two independent measurements, and the deviation was not greater than 6\%. Tre6P, Trehalose 6-phosphate; glc, glucose; G6P, glucose 6-phosphate.

\begin{tabular}{|c|c|c|c|c|c|c|c|c|}
\hline \multirow{2}{*}{ Kinetic parameter/biochemical property } & \multirow{2}{*}{ Concn $(\mathrm{mM})$} & \multicolumn{4}{|c|}{ OtsA } & \multirow[t]{2}{*}{ OtsB Tre6P } & \multicolumn{2}{|c|}{ TreS } \\
\hline & & UDP-glc & ADP-glc & GDP-glc & TDP-glc & & Maltose & Trehalose \\
\hline$K_{\mathrm{m}}(\mathrm{mM})$ & - & $0.3 \pm 0.05$ & $0.6 \pm 0.15$ & $0.4 \pm 0.1$ & $2.0 \pm 0.4$ & $0.7 \pm 0.1$ & $6.4 \pm 0.3$ & $58.9 \pm 3.0$ \\
\hline$K_{\mathrm{m}}(\mathrm{mM})$ for $\mathrm{G} 6 \mathrm{P}$ & - & $3.0 \pm 0.4$ & $4.2 \pm 0.7$ & $7.1 \pm 1.6$ & $5.3 \pm 0.8$ & - & - & - \\
\hline$V_{\max }\left[\mathrm{U}(\mathrm{mg} \text { protein })^{-1}\right]$ & - & $16.8 \pm 0.7$ & $14.2 \pm 0.8$ & $13.6 \pm 0.8$ & $30.0 \pm 1.2$ & $133.1 \pm 4.1$ & $47.9 \pm 0.8$ & $157.0 \pm 2.7$ \\
\hline $\mathrm{Mg}^{2+}$ & 0 & $\overline{84}$ & 85 & 84 & 83 & 3 & 100 & - \\
\hline $\mathrm{Mg}^{2+}$ & 10 & 100 & 100 & 100 & 100 & 100 & 88 & - \\
\hline $\mathrm{Mn}^{2+}$ & 10 & 54 & 69 & 63 & 58 & 5 & 90 & - \\
\hline $\mathrm{Ca}^{2+}$ & 10 & 54 & 63 & 51 & 41 & 0 & 100 & - \\
\hline $\mathrm{Co}^{2+}$ & 10 & 83 & 81 & 73 & 78 & 4 & 77 & - \\
\hline $\mathrm{Cu}^{2+}$ & 10 & 0 & 0 & 0 & 1 & 0 & 0 & - \\
\hline $\mathrm{NaCl}^{*}$ & 100 & 128 & 65 & 110 & 59 & 93 & 89 & - \\
\hline $\mathrm{KCl}^{*}$ & 100 & 99 & 56 & 90 & 45 & - & - & - \\
\hline
\end{tabular}

*The enzymic assay contained $10 \mathrm{mM} \mathrm{Mg}^{2+}$. 
Table 2. Amino acid sequence identity of OtsA, OtsB and TreS from $P$. freudenreichii $(P f)$ compared with known protein counterparts

The GenPept nos for OtsA, OtsB and Tres, respectively, are: YP_055672, YP_055673 and YP_055819 for P. acnes; NP_218007, NP_217889 and NP_214640 for M. tuberculosis; NP_601825, NP_601827 and NP_601502 for C. glutamicum; NP_825113, NP_825114 and NP_823979 for Streptomyces avermitilis; NP_302467 (OtsA) and NP_301392 (OtsB) for Mycobacterium lepra; NP_959507 (OtsA) and NP_962412 (OtsB) for Mycobacterium avium subsp. paratuberculosis; ZP_00659535 (OtsA) and ZP_00659540 (OtsB) for Nocardioides sp. JS614 and NP_739118 (OtsA) and NP_739120 (OtsB) for Corynebacterium efficiens.

\begin{tabular}{|lccc|}
\hline \multirow{2}{*}{ Organism } & \multicolumn{3}{c|}{ Sequence identity (\%) } \\
\cline { 2 - 4 } & Pf OtsA & Pf OtsB & Pf TreS \\
\hline P. acnes & 60 & 37 & 65 \\
Nocardioides sp. JS614 & 56 & 40 & - \\
M. avium subsp. paratuberculosis & 53 & 20 & - \\
M. leprae & 52 & 31 & - \\
C. efficiens & 51 & 23 & - \\
S. avermitilis & 33 & 42 & 58 \\
M. tuberculosis & 52 & 32 & 60 \\
C. glutamicum & 48 & 23 & 58 \\
\hline
\end{tabular}

Trehalose-6-phosphate phosphatase. The recombinant OtsB showed absolute substrate specificity for trehalose 6phosphate. OtsB activity was strictly dependent on $\mathrm{Mg}^{2+}$, and $50 \%$ of the maximum activity was reached with $0.1 \mathrm{mM} \mathrm{Mg}^{2+}$; the activity was abolished by addition of EDTA $(10 \mathrm{mM})$. The phosphatase activity was very low, or not detected, when $\mathrm{Mg}^{2+}$ was replaced by $\mathrm{Mn}^{2+}$, $\mathrm{Ca}^{2+}, \mathrm{Co}^{2+}$ or $\mathrm{Cu}^{2+}$ (Table 1$) . \mathrm{NaCl}$ had a slightly negative effect on OtsB activity in the range of $0-250 \mathrm{mM}$, but was clearly inhibitory (over $30 \%$ ) at higher concentrations. The activity was strongly inhibited $(70 \%)$ by $10 \mathrm{mM} \mathrm{NaF}$, a well-known phosphatase inhibitor. OtsB had maximal activity at $50^{\circ} \mathrm{C}$ and $\mathrm{pH} 6.5$ (Fig. 3). A hyperbolic dependence of the activity on the concentration of trehalose 6-phosphate was found. The kinetic parameters for OtsB are shown in Table 1.

Trehalose synthase. The presence of $\mathrm{Ca}^{2+}, \mathrm{Mg}^{2+}$, $\mathrm{Mn}^{2+}$ or $\mathrm{Co}^{2+}$ did not affect, or only slightly inhibited, TreS activity, while $\mathrm{Cu}^{2+}$ had a strong inhibitory effect (Table 1). Furthermore, EDTA $(10 \mathrm{mM})$ or $\mathrm{NaCl}$ $(100 \mathrm{mM})$ did not affect TreS activity, whereas Tris/HCl $(10 \mathrm{mM})$ had an inhibitory effect of $64 \%$. The temperature profile of TreS activity was investigated in the range of $30-60^{\circ} \mathrm{C}$ using maltose as the substrate; maximal activity was observed at $45^{\circ} \mathrm{C}$, and the optimal $\mathrm{pH}$ was 5.2 (Fig. 3). The $K_{\mathrm{m}}$ for maltose was $6.4 \mathrm{mM}$, which was nine times lower than that for trehalose (Table 1).
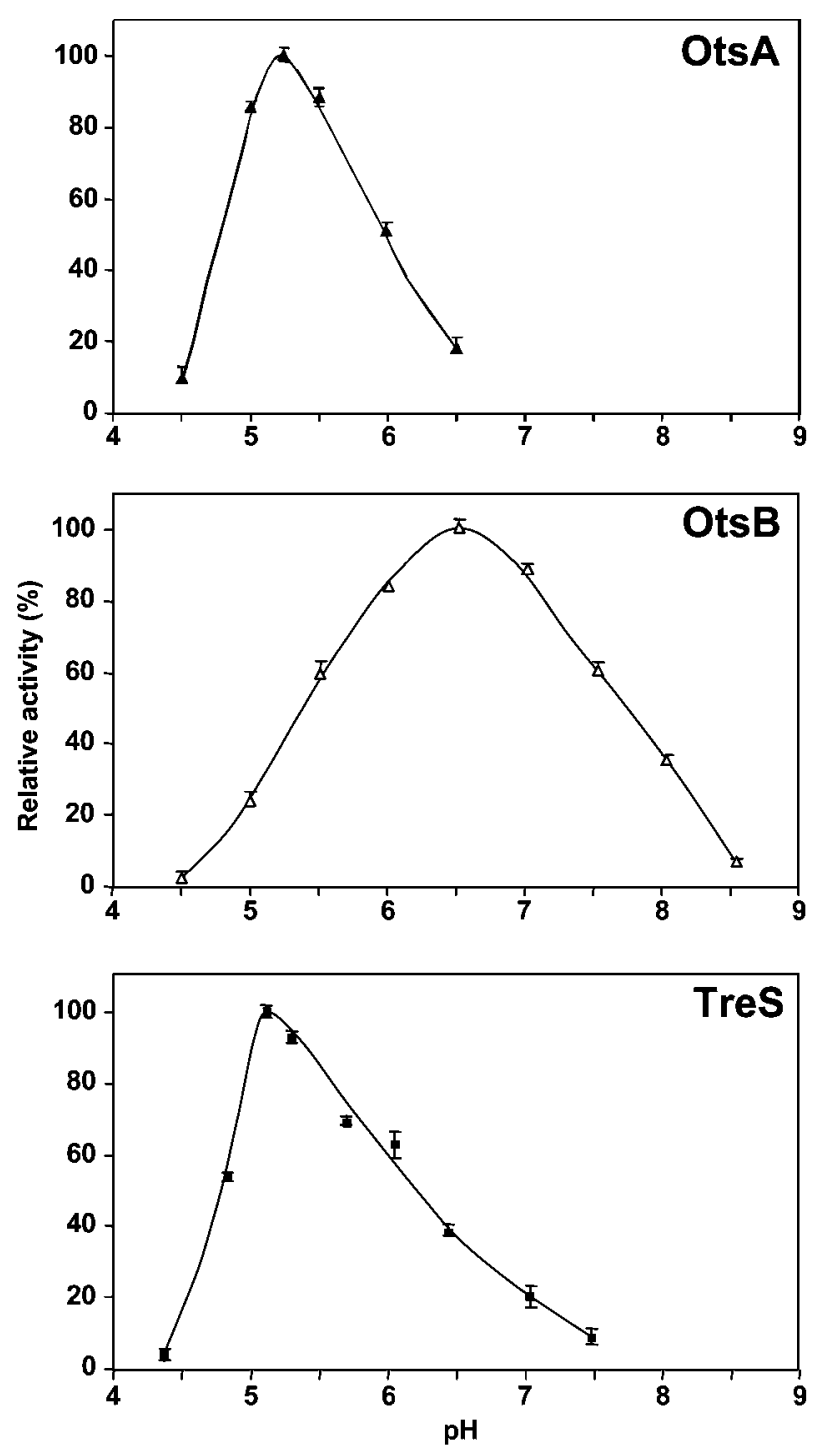

Fig. 3. Effect of $\mathrm{pH}$ on the activity of OtsA, OtsB and TreS. The OtsA activity was determined with TDP-glucose and glucose 6-phosphate as substrates; the activity of TreS was determined using maltose. Error bars indicate SD.

\section{Modulation of the substrate specificity of trehalose-6-phosphate synthase by component(s) of the cell extract}

OtsA activity in $P$. freudenreichii extracts was observed only when ADP-glucose was the glucosyl donor substrate, whereas recombinant OtsA showed activity with ADP-, GDP-, UDP- and TDP-glucose. To investigate this discrepant behaviour, the native OtsA was partially purified. After the third purification step, the initial specificity of OtsA for ADP-glucose was lost (Fig. 4). Surprisingly, the recombinant protein became specific for ADP-glucose upon incubation with a dialysed $P$. freudenreichii extract (Fig. 4). It appeared that the cell extract contained one or more components that interacted with the protein, and induced a 
(a)

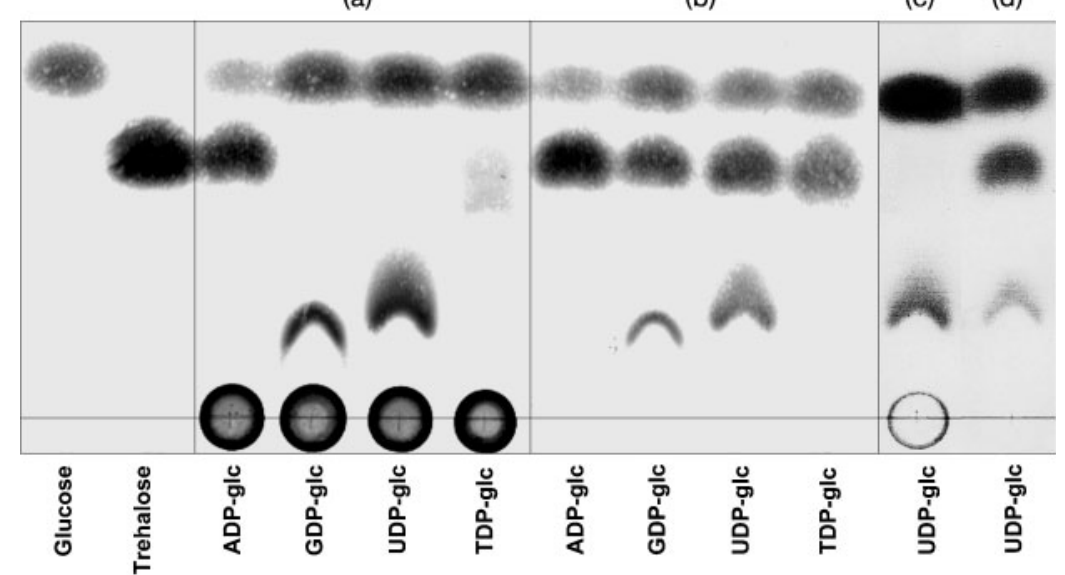

Fig. 4. Differential substrate specificity of OtsA in cell extracts and purified preparations. TLC analysis of reaction products: (a) native OtsA in a dialysed cell extract, and (b) partially purified OtsA with different glucosyl donors; and recombinant OtsA without (c) and with (d) addition of a dialysed extract of $P$. freudenreichii. Details of the reaction mixtures and incubations are described in Methods. clear change in the substrate specificity of OtsA. To check whether OtsB could be responsible for this effect, we examined the substrate specificity of recombinant OtsA in the presence of pure recombinant OtsB (OtsA:OtsB, molar ratio $1.8: 1)$. The substrate preference of OtsA was not affected by the presence of OtsB (data not shown).

\section{Levels of OtsA and TreS in response to acid, oxidative and osmotic stress}

To obtain insight into the physiological role of the two pathways identified in $P$. freudenreichii for trehalose metabolism, immunoblotting assays were used to assess the levels of OtsA and TreS during acid stress ( $\mathrm{pH}$ downshift from 7 to 5), oxidative stress (oxygen concentration $0.115 \mathrm{mM}$ ) and osmotic stress [increase of $\mathrm{NaCl}$ in the medium from 0 to $2 \%(\mathrm{w} / \mathrm{v})]$. Stress submission started at a time designated zero. Constitutive levels of OtsA and TreS were detected under optimal growth conditions (Fig. 5). The amount of OtsA increased approximately twofold when any of the three stresses were imposed from 2 to $10 \mathrm{~h}$. In contrast, TreS decreased to about one-quarter of the initial level (acid or oxidative stress, from 0 to $5 \mathrm{~h}$ ), or did not change significantly (osmotic stress) (Fig. 5). The effect of these stresses on the intracellular level of trehalose was assessed in the same experiments. Trehalose content increased notably (around sevenfold), from a basal level of $30 \mathrm{mg}$ trehalose (g protein) ${ }^{-1}$ to approximately $200 \mathrm{mg}$ trehalose $(\mathrm{g} \text { protein })^{-1}$ during the initial $5 \mathrm{~h}$ period in response to oxidative stress and osmotic stress. In cells subjected to acid stress, the level of trehalose increased 2.3fold only, to around $70 \mathrm{mg}$ trehalose (g protein) $)^{-1}$ (data not shown).

\section{DISCUSSION}

In the present work, the pathways for trehalose metabolism in $P$. freudenreichii were characterized, and their roles in response to osmotic, oxidative and acid stress were examined. As the genome sequence of this organism is not available ( $P$. acnes is the only species of the genus Propionibacterium whose genome sequence is known), relevant activities were sought in cell extracts, and the respective genes obtained using degenerate primers and inverse PCR. Two pathways for trehalose metabolism were found in P. freudenreichii: the OtsA-OtsB pathway, used for

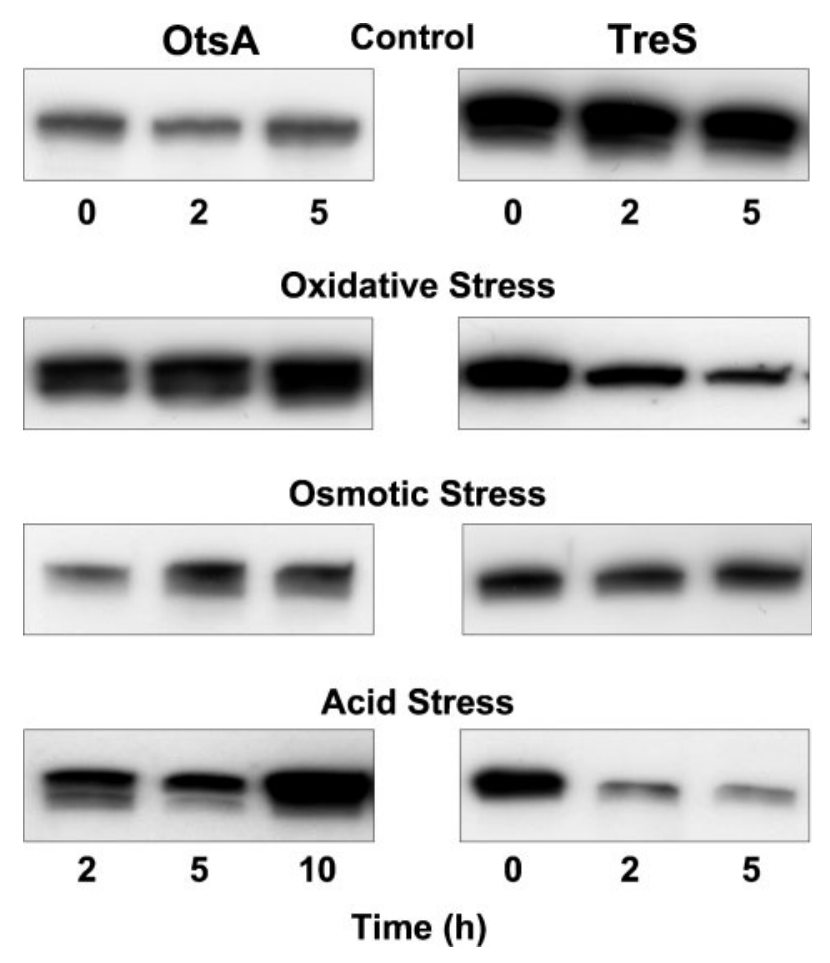

Fig. 5. Immunoblotting assays of OtsA and TreS in P. freudenreichii cells subjected to oxidative, osmotic and acid stress. Cells grown at optimal temperature and $\mathrm{pH}$ were subjected to oxidative stress (oxygen concentration $0.115 \mathrm{mM}$ ), osmotic stress $[2 \%(w / v) ~ N a C l]$ and acid stress ( $\mathrm{pH}$ shift to 5$)$. Cell samples were withdrawn at the times indicated, and the levels of OtsA and TreS were assessed by immunoblotting. Time zero corresponds to cells immediately before stress imposition. Each lane contains $5 \mu \mathrm{g}$ total protein. Each experiment was done at least in triplicate with samples from a single growth for each condition. 
trehalose synthesis, and the TreS pathway. The majority of organisms examined use the OtsA-OtsB pathway for trehalose synthesis, and trehalase, or other hydrolytic activities, for the catabolism of this disaccharide. We showed that $P$. freudenreichii does not possess any of these hydrolytic activities, and propose that TreS is used to accomplish the catabolic function.

The genes and enzymes involved in the OtsA-OtsB and the TreS pathways were characterized in detail. The organization of the ots $A$ and ots $B$ genes is similar to that of $P$. acnes, insofar as the otsB gene is found immediately upstream of the otsA gene in the other strand. A different organization was found in Mycobacterium tuberculosis and Corynebacterium glutamicum, where the genes are found in the same strand, and are separated by genes unrelated to trehalose metabolism (Cole et al., 1998; Kalinowski et al., 2003). Interestingly, in $P$. freudenreichii, genes encoding enzymes for the synthesis of vitamin $B_{12}$ were found immediately downstream of the OtsA-OtsB operon-like-structure, while homofunctional genes have been found elsewhere in the genome of $P$. acnes (Brüggemann et al., 2004). In $P$. freudenreichii, as in $P$. acnes, treS is flanked by genes encoding a putative $\alpha$-amylase and a hypothetical protein involved in trehalose synthesis, and the otsA/otsB genes are far away from treS. In contrast, in the bacterium Thermus thermophilus, the genes encoding the OtsA-OtsB and TreS pathways are contiguous, and organized in a single operonlike structure (Silva et al., 2003).

Trehalose-6-phosphate synthase has been characterized in a number of different organisms, including bacteria, yeast, fungi, insects and plants (Elbein et al., 2003). Probably the most interesting feature in the comparative enzymology of OtsA proteins is the diverse specificity with regard to the glucosyl donor. In cell extracts, the OtsA of $P$. freudenreichii was absolutely specific for ADP-glucose, a finding that appears to be unique as far as OtsA proteins are concerned. In fact, known OtsA proteins either can use the four glucosyl donors (Lapp et al., 1971; Killick, 1979; Pan et al., 2002; Silva et al., 2005), or are specific for UDP- or GDPglucose (Elbein, 1968; Giæver et al., 1988). Most surprising was the observation that the pure (native or recombinant) OtsA of $P$. freudenreichii lacked the absolute specificity shown in cell extracts, and recognized any of the four sugar-nucleotides as a substrate. Remarkably, the recombinant OtsA became specific to ADP-glucose when added to a dialysed extract of $P$. freudenreichii, reproducing the behaviour of native OtsA in cell extracts. These results suggest the presence of a regulatory protein, or interaction with other component(s) of the intracellular milieu. Indeed, in Saccharomyces cerevisiae, trehalose-6-phosphate synthase (TPS1) is part of a multimeric complex comprising trehalose-6-phosphate phosphatase (TPS2) and two putative regulatory proteins (Reinders et al., 1997). This led us to think that OtsB could be the protein responsible for the observed change in the substrate specificity of pure OtsA. The influence of purified OtsB on the specificity of OtsA was examined, but no change was observed. The reason for such dramatic extract-dependent change in substrate specificity remains unclear, but an interaction with a regulatory component appears to be a plausible explanation.

We found that $P$. freudenreichii cells responded to acid, oxidative and osmotic stress by selectively enhancing the levels of the synthase of the OtsA-OtsB pathway, while the level of the enzyme of the TreS pathway remained constant or decreased. These data support the view that, under stress conditions, trehalose is predominantly produced via the OtsA-OtsB pathway. On the other hand, the fact that the level of TreS decreased in response to the different stresses downplays the importance of the TreS pathway in trehalose synthesis under stress. In addition, the high content of intracellular trehalose [up to $0.4 \mathrm{~g}$ (g cell protein) ${ }^{-1}$ ], and undetectable levels of maltose, probably leave trehalose as the sole substrate available for TreS. One could argue that the intracellular maltose concentrations would reach higher levels during maltose consumption, but this hypothesis could not be tested, since $P$. freudenreichii was unable to grow on this substrate. These findings, together with the absence in $P$. freudenreichii extracts of other activities commonly involved in trehalose degradation, led to the proposal that TreS is responsible for trehalose degradation, which proceeds through maltose, the reaction product. As maltose phosphorylase has been reported in a different strain of $P$. freudenreichii (Aisaka et al., 1996), several attempts were made to detect this activity, but all the assays produced negative results. On the other hand, a strong activity of amylomaltase was detected in $P$. freudenreichii extracts: maltose was a substrate for amylomaltase in the presence of trace amounts of maltooligosaccharides. Therefore, maltose derived from TreS activity can be processed by amylomaltase, releasing glucose from the reducing end of maltooligosaccharides. The resulting glucose is phosphorylated by the activity of polyphosphateand ATP-dependent glucokinase present in cells of $P$. freudenreichii (Phillips et al., 1993; Pereira, 1997), and is further catabolized via glycolysis (Fig. 6).

This work led to the proposal that $P$. freudenreichii uses the OtsA-OtsB pathway for trehalose synthesis, whereas trehalose catabolism proceeds via TreS. The physiological role of the multiple pathways associated with trehalose metabolism has been unequivocally established in a few bacteria only (De Smet et al., 2000; Wolf et al., 2003; Makihara et al., 2005). Firm evidence for the role of TreS in trehalose degradation has been obtained with mutants of Rhodobacter sphaeroides (Makihara et al., 2005), and a similar function for TreS has been proposed in $C$. glutamicum. In the latter organism, the reaction catalysed by TreS proceeds in the direction of trehalose synthesis only when maltose is the carbon source for growth (Wolf et al., 2003). The $K_{\mathrm{m}}$ values for trehalose of known TreS proteins are in the range of $100 \mathrm{mM}$, which is about one order of magnitude greater than those for maltose; therefore, TreS appears ideally suited to act upon trehalose in organisms 


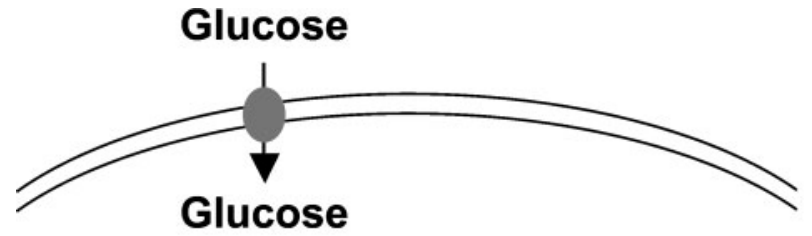

1 PolyP

Glucose 6-phosphate

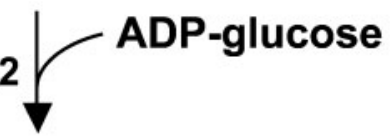

Trehalose 6-phosphate

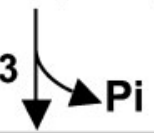

TREHALOSE

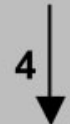

Maltose

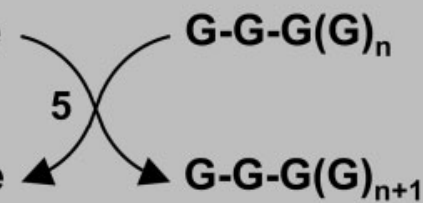

Glucose

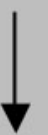

Glycolysis

Fig. 6. Proposed scheme of trehalose metabolism in $P$. freudenreichii. 1, Glucokinase; 2, trehalose-6-phosphate synthase; 3, trehalose-6-phosphate phosphatase; 4, trehalose synthase; 5, amylomaltose; PolyP, polyphosphate; G, glucose.

that accumulate high levels of this disaccharide as a compatible solute or carbon reserve.

BLAST searches of the protein databases with the sequences of $P$. freudenreichii TreS and OtsA revealed an enormous disparity in the number of hits for the two enzymes, highlighting the frequent occurrence of OtsA, and the scarce distribution of TreS. The common OtsA-OtsB pathway is generally assumed to be involved in the synthesis of trehalose, but, for example, in C. glutamicum it has been shown that the mutant with a deletion of ots $A$ accumulates levels of trehalose comparable with those of the wild-type under identical osmotic stress conditions, with the synthesis proceeding via the TreY-TreZ pathway (Wolf et al., 2003). In $R$. sphaeroides, the OtsA-OtsB pathway is the major route for trehalose synthesis under osmotic stress, and the second pathway, TreY-TreZ, contributes to a minor extent (Makihara et al., 2005).

The regulation of the multiple pathways in response to stress other than osmotic stress has not been reported, but herein we show that the enhancement of the OtsA-OtsB pathway in $P$. freudenreichii is a response common to all the three stresses imposed. Trehalose accumulation was stimulated in response to osmotic, oxidative and acid stress, suggesting a role of this solute in cell protection against these diverse stresses. Trehalose is a canonical osmolyte, accumulating to high levels to preserve cell turgor in environments of elevated osmolarity (Argüelles, 2000; Elbein et al., 2003). Recent studies, however, have demonstrated the ability of this disaccharide to protect cells against other types of aggressions, namely oxidative stress and heat stress. The role of trehalose as a scavenger of oxygen radicals has been shown in S. cerevisiae (Benaroudj et al., 2001). On the other hand, the accumulation of trehalose in response to elevated temperature has been reported in several mesophilic organisms, such as yeast, E. coli and Salmonella enterica (Strom \& Kaasen, 1993; De Virgilio et al., 1994; Cánovas et al., 2001). Protection against acid stress has not been reported; however, it is interesting that genes for the synthesis of trehalose are present in the genome of Picrophilus torridus, an extreme acidophile with optimal $\mathrm{pH}$ for growth of 0.7 (Fütterer et al., 2004). It is conceivable that trehalose plays a role in the stabilization of cell membranes, which must withstand a strong $\mathrm{pH}$ gradient in such acidic environments.

Herein, a global picture of trehalose metabolism in $P$. freudenreichii was obtained regarding adaptation to osmotic, oxidative and acid stress. Under all types of stress examined, the expression of the OtsA-OtsB pathway for trehalose synthesis was clearly enhanced. The experimental data presented here strongly suggest the involvement of the TreS pathway in trehalose catabolism; final proof, however, was not obtained due to lack of mutants. These findings represent an important contribution to our understanding of stress responses in propionibacteria with respect to trehalose synthesis, and this knowledge is essential to the design of dairy strains optimized for the in situ production of trehalose, an efficient flavour preservative of fermented foods. However, further progress in this area of research must await the development of genetic tools for the manipulation of $P$. freudenreichii.

\section{ACKNOWLEDGEMENTS}

This work was supported by the EC, contract number QLK1-CT-200001376, and by Fundação para a Ciência e a Tecnologia (FCT) and FEDER (project POCTI/BIO/48333/2002). F. S. C. and N. B. acknowledge FCT for the award of research fellowships (SFRH/BD/5080/2001 and SFRH/BPD/14841/2003). Propionibacterium NIZO B365 was kindly supplied by J. Hugenholtz, NIZO. We are grateful to A. Ramos for help during the initial phase of the work, to C. Jorge for supplying $R$. marinus trehalase, and to A. Mingote for technical assistance. 


\section{REFERENCES}

Aisaka, K., Masuda, T. \& Chikamune, T. (1996). Properties of maltose phosphorylase from Propionibacterium freudenreichii. J Ferment Bioeng 82, 171-173.

Ames, B. N. (1966). Assay of inorganic phosphate, total phosphate and phosphatases. Methods Enzymol 8, 115-118.

Andersson, U., Levander, F. \& Rådström, P. (2001). Trehalose-6phosphate phosphorylase is part of a novel metabolic pathway for trehalose utilization in Lactococcus lactis. J Biol Chem $\mathbf{2 7 6}$ 42707-42713.

Argüelles, J. C. (2000). Physiological roles of trehalose in bacteria and yeasts: a comparative analysis. Arch Microbiol 174, 217-224; erratum 174, 456.

Belocopitow, E. \& Maréchal, L. R. (1970). Trehalose phosphorylase from Euglena gracilis. Biochim Biophys Acta 198, 151-154.

Benaroudj, N., Lee, D. H. \& Goldberg, A. L. (2001). Trehalose accumulation during cellular stress protects cells and cellular proteins from damage by oxygen radicals. J Biol Chem 276, 24261-24267.

Brüggemann, H., Henne, A., Hoster, F., Liesegang, H., Wiezer, A., Strittmatter, A., Hujer, S., Durre, P. \& Gottschalk, G. (2004). The complete genome sequence of Propionibacterium acnes, a commensal of human skin. Science 305, 671-673.

Cánovas, D., Fletcher, S. A., Hayashi, M. \& Csonka, L. N. (2001). Role of trehalose in growth at high temperature of Salmonella enterica serovar Typhimurium. J Bacteriol 183, 3365-3371.

Cardoso, F. S., Gaspar, P., Hughenholtz, J., Ramos, A. \& Santos, H. (2004). Enhancement of trehalose production in dairy propionibacteria through manipulation of environmental conditions. Int J Food Microbiol 91, 195-204.

Cole, S. T., Brosch, R., Parkhill, J., Garnier, T., Churcher, C., Harris, D., Gordon, S. V., Eiglmeier, K., Gas, S. other authors (1998). Deciphering the biology of Mycobacterium tuberculosis from the complete genome sequence. Nature 393, 537-544; erratum 396, 190.

da Costa, M. S., Santos, H. \& Galinski, E. A. (1998). An overview of the role and diversity of compatible solutes in Bacteria and Archaea. Adv Biochem Eng Biotechnol 61, 117-153.

De Smet, K. A., Weston, A., Brown, I. N., Young, D. B. \& Robertson, B. D. (2000). Three pathways for trehalose biosynthesis in mycobacteria. Microbiology 146, 199-208.

De Virgilio, C., Hottiger, T., Dominguez, J., Boller, T. \& Wiemken, A. (1994). The role of trehalose synthesis for the acquisition of thermotolerance in yeast. I. Genetic evidence that trehalose is a thermoprotectant. Eur J Biochem 219, 179-186.

Elbein, A. D. (1968). Trehalose phosphate synthesis in Streptomyces hygroscopicus: purification of guanosine diphosphate D-glucose: D-glucose-6-phosphate 1-glucosyl-transferase. J Bacteriol 96, 1623-1631.

Elbein, A. D. Y. T., Pan, Y. T., Pastuszak, I. \& Carroll, D. (2003). New insights on trehalose: a multifunctional molecule. Glycobiology 13, 17R-27R.

Fütterer, O., Angelov, A., Liesegang, H., Gottschalk, G., Schleper, C., Schepers, B., Dock, C., Antranikian, G. \& Liebl, W. (2004). Genome sequence of Picrophilus torridus and its implications for life around pH 0. Proc Natl Acad Sci U S A 101, 9091-9096.

Giæver, H. M., Styrvold, O. B., Kaasen, I. \& Strom, A. R. (1988). Biochemical and genetic characterization of osmoregulatory trehalose synthesis in Escherichia coli. J Bacteriol 170, 2841-2849.

Horlacher, R., Uhland, K., Klein, W., Ehrmann, M. \& Boos, W. (1996). Characterization of a cytoplasmic trehalase of Escherichia coli. J Bacteriol 178, 6250-6257.
Huang, Y. \& Adams, M. C. (2004). In vitro assessment of the upper gastrointestinal tolerance of potential probiotic dairy propionibacteria. Int J Food Microbiol 91, 253-260.

Jan, G., Belzacq, A. S., Haouzi, D., Rouault, A., Metivier, D., Kroemer, G. \& Brenner, C. (2002). Propionibacteria induce apoptosis of colorectal carcinoma cells via short-chain fatty acids acting on mitochondria. Cell Death Differ 9, 179-188.

Kalinowski, J., Bathe, B., Bartels, D. \& 24 other authors (2003). The complete Corynebacterium glutamicum ATCC 13032 genome sequence and its impact on the production of L-aspartate-derived amino acids and vitamins. J Biotechnol 104, 5-25.

Killick, K. A. (1979). Trehalose-6-phosphate synthase from Dictyostelium discoideum: partial purification and characterization of the enzyme from young sorocarps. Arch Biochem Biophys 196, 121-133.

Lapp, D., Patterson, B. W. \& Elbein, A. D. (1971). Properties of a trehalose phosphate synthase from Mycobacterium smegmatis. J Biol Chem 246, 4567-4579.

Makihara, F., Tsuzuki, M., Sato, K., Masuda, S., Nagashima, K. V., Abo, M. \& Okubo, A. (2005). Role of trehalose synthesis pathways in salt tolerance mechanism of Rhodobacter sphaeroides f. sp. denitrificans IL106. Arch Microbiol 184, 56-65.

Maréchal, L. R. \& Belocopitow, E. (1972). Metabolism of trehalose in Euglena gracilis. I. Partial purification and some properties of trehalose phosphorylase. J Biol Chem 247, 3223-3228.

Maruta, K., Nakada, T., Kubota, M., Chaen, H., Sugimoto, T., Kurimoto, M. \& Tsujisaka, Y. (1995). Formation of trehalose from maltooligosaccharides by a novel enzymatic system. Biosci Biotechnol Biochem 59, 1829-1834.

Nakada, T., Maruta, K., Tsusaki, K., Kubota, M., Chaen, H., Sugimoto, T., Kurimoto, M. \& Tsujisaka, Y. (1995). Purification and properties of a novel enzyme, maltooligosyl trehalose synthase, from Arthrobacter sp. Q36. Biosci Biotechnol Biochem 59, 2210-2214.

Nishimoto, T., Nakano, M., Nakada, T., Chaen, H., Fukuda, S., Sugimoto, T., Kurimoto, M. \& Tsujisaka, Y. (1996). Purification and properties of a novel enzyme, trehalose synthase, from Pimelobacter sp. R48. Biosci Biotechnol Biochem 60, 640-644.

Pan, Y. T., Carroll, J. D. \& Elbein, A. D. (2002). Trehalose-phosphate synthase of Mycobacterium tuberculosis. Cloning, expression and properties of the recombinant enzyme. Eur J Biochem 269, 6091-6100.

Pereira, H. (1997). The relationship between carbon and phosphorous metabolism of polyphosphate-accumulating bacteria: in vivo NMR studies. PhD thesis, New University of Lisbon, Lisbon, Portugal.

Phillips, N. F., Horn, P. J. \& Wood, H. G. (1993). The polyphosphateand ATP-dependent glucokinase from Propionibacterium shermanii: both activities are catalyzed by the same protein. Arch Biochem Biophys 300, 309-319.

Qu, Q., Lee, S.-J. \& Boos, W. (2004). TreT, a novel trehalose glycosyltransferring synthase of the hyperthermophilic archaeon Thermococcus litoralis. J Biol Chem 279, 47890-47897.

Reinders, A., Burckert, N., Hohmann, S., Thevelein, J. M., Boller, T., Wiemken, A. \& De Virgilio, C. (1997). Structural analysis of the subunits of the trehalose-6-phosphate synthase/phosphatase complex in Saccharomyces cerevisiae and their function during heat shock. Mol Microbiol 24, 687-695.

Rimmele, M. \& Boos, W. (1994). Trehalose-6-phosphate hydrolase of Escherichia coli. J Bacteriol 176, 5654-5664.

Roessner, C. A., Huang, K. X., Warren, M. J., Raux, E. \& Scott, A. I. (2002). Isolation and characterization of 14 additional genes specifying the anaerobic biosynthesis of cobalamin (vitamin $\mathrm{B}_{12}$ ) in Propionibacterium freudenreichii (P. shermanii). Microbiology 148, 1845-1853. 
Sambrook, J., Fritsch, E. F. \& Maniatis, T. (1989). Molecular cloning: a Laboratory Manual, 2nd edn. Cold Spring Harbor, NY: Cold Spring Harbor Laboratory.

Santos, H. \& da Costa, M. S. (2001). Organic solutes from thermophiles and hyperthermophiles. Methods Enzymol 334, 302-315.

Silva, Z., Alarico, S., Nobre, A., Horlacher, R., Marugg, J., Boos, W., Mingote, A. I. \& da Costa, M. S. (2003). Osmotic adaptation of Thermus thermophilus RQ-1: lesson from a mutant deficient in synthesis of trehalose. J Bacteriol 185, 5943-5952.

Silva, Z., Alarico, S. \& da Costa, M. S. (2005). Trehalose biosynthesis in Thermus thermophilus RQ-1: biochemical properties of the trehalose-6-phosphate synthase and trehalose-6-phosphate phosphatase. Extremophiles 9, 29-36.

Smith, D. E. \& Fisher, P. A. (1984). Identification, developmental regulation, and response to heat shock of two antigenically related forms of a major nuclear envelope protein in Drosophila embryos: application of an improved method for affinity purification of antibodies using polypeptides immobilized on nitrocellulose blots. J Cell Biol 99, 20-28.

Stjernholm, R. (1958). Formation of trehalose during dissimilation of glucose by Propionibacterium. Acta Chem Scan 12, 646-649.

Strom, A. R. \& Kaasen, I. (1993). Trehalose metabolism in Escherichia coli: stress protection and stress regulation of gene expression. Mol Microbiol 8, 205-210.

Uhland, K., Mondigler, M., Spiess, C., Prinz, W. \& Ehrmann, M. (2000). Determinants of translocation and folding of TreF, a trehalase of Escherichia coli. J Biol Chem 275, 23439-23445.

Wolf, A., Krämer, R. \& Morbach, S. (2003). Three pathways for trehalose metabolism in Corynebacterium glutamicum ATCC 13032 and their significance in response to osmotic stress. Mol Microbiol 49, 1119-1134.

Edited by: R. W. Hutkins 